Cross modal effect of nat al habitat experi ence i ncr eases recept i vi ty to non- nat al habi tat cues in general ist parasi tic wasps

\begin{tabular}{|l|l|}
\hline 著者 & Takentot o H r oyuki \\
\hline $\begin{array}{l}\text { j our nal or } \\
\text { publ i cat i on t i t l e }\end{array}$ & Ar thr opod- pl ant i nt er act i ons \\
\hline vol une & 10 \\
\hline nunber & 4 \\
\hline page r ange & 303 - 310 \\
\hline year & $2016-04$ - 26 \\
\hline 出版者 & Spr i nger Nat ur e \\
\hline URL & ht t p: //hdl . handl e. net /10297/10108 \\
\hline
\end{tabular}




\title{
Cross modal effect of natal habitat experience increases receptivity to non-natal habitat cues in generalist parasitic wasps
}

\author{
Hiroyuki Takemoto ${ }^{1, *}$ \\ ${ }^{1}$ Research Institute of Green Science and Technology, Shizuoka University; Oya 836, Shizuoka, 422-8529, Japan \\ *corresponding author email: semio.chem.ecol@shizuoka.ac.jp Tel +81542384834
}

\begin{abstract}
Exposure to natal habitats induces preferences in individual organism for foraging cues that originate from their developmental habitat. However, the natal experience of habitat generalists may play a different role in their habitat selection, since they use a broad range of foraging cues in a non-natal habitat. In this study, the effects of natal habitat experiences on the responses of females of the generalist parasitoid Aphidius gifuensis Ashmead to cues from natal or non-natal habitats were investigated. The landing rates on plant-host complexes (PHCs) and undamaged plants (UDPs) of natal (wheat) and non-natal (broad bean) plant systems in a wind tunnel increased after encounters with host aphids on the wheat-PHCs when the wasps had previously been exposed to wheat-UDPs. However, without the previous exposure to wheat-UDPs, the landing rate on the broad bean-PHCs was not increased. Similar increase found in responses to green paper disk dummies suggested involvement of visual stimuli in the reinforcement. In olfactometer tests, preferences for broad bean-UDPs over broad bean-PHCs were found but wasps with previous exposure to wheat-UDPs and host-encounter on wheat-PHCs did not show the preference. These results suggest that the early exposure to natal habitat plants modified later learning and response to visual cue from potential non-natal habitat plants, and olfactory learning might be involved in the modification. This cross modal effect of early natal experience would compensate for the loss of host searching efficiency due to uncertain information of potential non-natal habitats.
\end{abstract}

Keywords habitat selection; natal experience; learning; host preference; olfaction; vision

\section{Introduction}

The recognition of environmental stimuli by individual organisms is deeply involved in their foraging efficiency for foods, hosts and habitats. Factors involved in the efficient responses to foraging cues need to be determined to better understand the mechanisms responsible for biological interactions. Animals of various taxa acquire preferences for stimuli originating from their natal habitat (Davis and Stamps 2004). Populations of animals that experienced a suitable habitat are likely to specialize on the particular habitat but the range of resources used by these animals are often broad because of resource variability. Habitat generalists, which use a broad range of resources, disperse and move from the natal habitat to non-natal habitats over the life time of the individuals. The host locational process of habitat generalists should include a step in which they respond to foraging cues from the new, possible habitats. In that step, the availability of cues from non-natal habitats is important to increase their foraging efficiency. I investigate how the experience in natal habitats by generalists influences their responses to cues originating from non-natal habitats.

Parasitic wasps use environmental stimuli from multiple sensory modalities, including vision and olfaction, as cues to their host location (Godfray 1993; Turlings et al. 1993; Vet and Dicke 1992; Vet et al. 1995). The natal habitat preference induction of parasitic wasps has been most frequently observed among animal taxonomic groups (Davis and Stamps 2004). Parasitic wasps can change their responses to host locational cues after an encounter with an unconditioned stimuli derived from the hosts themselves. The changes are possibly due to the sensitization to general stimuli, or associative learning with specific conditioned stimuli being associated with the host encounter (Vet et al. 1995). Although host encounter experiences have the highest impact on the behavioural changes of host-searching female wasps, experiences prior to the host encounter can be direct and indirect influences. 
The way experiences influence the response to host locational cues varies in terms of the events in an individual life time and of the sensory modalities involved. Olfactory preferences are acquired for odours from plants exposed during mummification in Aphidius rhopalosiphi de Stefani-Perez (Hymenoptera: Braconidae) (van Emden et al. 2002), and at emergence in Aphidius colemani Viereck (Hymenoptera: Braconidae) (Storeck et al. 2000) and Diaeretiella rapae McIntosh (Hymenoptera: Braconidae) (Blande et al. 2007). The Japanese strain of Aphidius ervi Haliday (Hymenoptera: Braconidae) acquires olfactory preferences through a two-steps experience: female wasps show olfactory responses to host-infested plants after they are exposed to the odour at emergence if she previously was exposed to the odour during mummification (Takemoto et al. 2012). The mechanisms and ecological significance of plural experiences at different developmental stages of parasitic wasps are mostly unknown. Information from olfactory and visual stimuli are integrated and used as foraging cues (Chittka and Raine 2006; Dötterl and Vereecken 2010). Experience can modify the integration of information from multisensory stimuli in generalist bees, and, in general, experienced bees are more likely to use visual cues, whereas inexperienced ones rely more on olfactory cues (Dobson 1994; Dötterl and Vereecken 2010).

The generalist parasitic wasp Aphidius gifuensis Ashmead (Hymenoptera: Braconidae) was used in this study. This species occurs in eastern Asia, including Japan (Takada 1992), and attacks aphid species of different taxa in Aphididae including Myzus persicae Sulzer (Hemiptera: Aphididae), Macrosiphum euphorbiae Thomas (Hemiptera: Aphididae), Aulacorthum solani Kaltenbach (Hemiptera: Aphididae), Acyrthosiphon pisum Harris (Hemiptera: Aphididae) and Sitobion akebiae Shinji (Hemiptera: Aphididae) (Ohta and Honda 2010; Takada 2002). Biological control studies using this species have accumulated (e.g. Ohta and Ohtaishi 2004; Ohta and Ohtaishi 2005; Wei et al. 2003; Yang et al. 2009b) as have their applications with companion plants (Nakahira et al. 2012; Ohta and Honda 2010). Because it is empirically clear that females of A. gifuensis move from natal habitats to non-natal habitats, the host-finding process of this species should include a step in which they respond to cues from the non-natal habitat.

In this study, I investigated whether the exposure of A. gifuensis female wasps to natal habitat plants (wheat) prior to host encounters in the natal habitat plants influences their later responses to olfactory and visual cues from non-natal habitat plants (broad bean), focusing on the exposure of $A$. gifuensis female wasps to natal habitat plants (wheat) prior to a host encounter as the conditioning treatment.

\section{Materials and methods}

Plants and insects

Four grams of wheat seeds (Triticum aestivum Linnaeus cv Norin 61) (Poaceae) were germinated on vermiculite in a vinyl cap $(\sim 300 \mathrm{ml})$, and grown in a climate controlled room $\left(22 \pm 2^{\circ} \mathrm{C}, 14 \mathrm{~L}: 8 \mathrm{D}, 20-40 \% \mathrm{RH}\right)$. At 10 to 14 days after germination, they were used for experimentation and insect rearing. Broad bean plants (Vicia faba Linnaeus cv Nintoku Issun) (Fabaceae) were individually grown in a vinyl cup $(\sim 300 \mathrm{ml})$ in a climate controlled room $\left(22 \pm 2{ }^{\circ} \mathrm{C}\right.$, 14L:8D, 20-40\% RH). Three weeks after germination, bean seedlings with two or three pairs of extended leaves were used for experimentation and insect rearing.

A colony of A. gifuensis was provided by I. Ohta (NARO Institute of Vegetable and Tea Science, Japan) in 2012, and maintained on $S$. akebiae on wheat. The S. akebiae colony was also provided by I. Ohta. To prepare the wasps, 100 adult $S$. akebiae aphids were transferred on to a pot of wheat plants, and 3 days after the transfer, the plant-host complex (PHC) was exposed to 30 female parasitoids. Then, 10 days after the parasitism, mummies were gently collected using forceps and used for the conditioning treatments in the experiments. An alternative host aphid for preparation of a non-natal habitat, pea aphid A. pisum was collected in Shizuoka, Japan, and maintained on broad bean plants. Insects were maintained in a climate controlled room under the same conditions mentioned above for plant maintenance $\left(22 \pm 2{ }^{\circ} \mathrm{C}, 14 \mathrm{~L}: 8 \mathrm{D}, 20-40 \% \mathrm{RH}\right)$.

\section{Conditioning procedure for parasitic wasps}

Four conditioning treatments of female wasps were prepared (Fig. 1), in which their host-encounter experiences and the period from emergence to host-encounter had two treatments each. A Petri dish containing mummies was placed in a plastic cage $(\mathrm{W} 365 \mathrm{~mm} \times \mathrm{D} 215 \mathrm{~mm} \times \mathrm{H} 250 \mathrm{~mm}$ ) with either a pot of undamaged (intact) wheat plants or wet paper towels only, without a plant. After the emergence of adult wasps, 120 female wasps were collected and 
transferred to another plastic cage of the same size containing either two pots of wheat plants, which had been infested by 100 alate adults of S. akebiae for 3 days (plant-host complex; PHC), or wet paper towels only, without PHCs. Then, 30 min after the transfer, wasps on PHCs were collected and kept in another plastic cage of the same size containing wet paper towels. These treatments of wasps were referred to as N-N, N-E, E-N and E-E, respectively, in which E referred to exposure to plants or PHCs and N referred to no exposure to plants or PHCs. Wasps were fed a 50\% (w/vol) aqueous honey solution ad libitum.

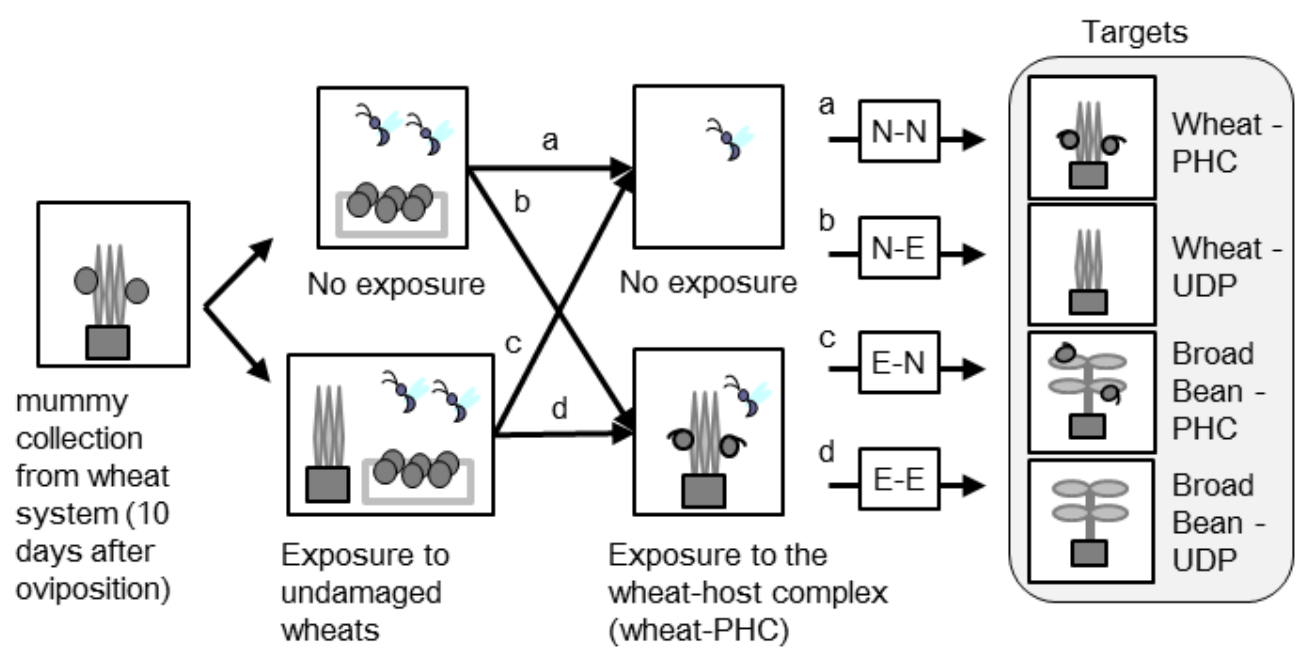

Fig. 1 Conditioning procedures for wasps in Experiments 1, 2 and 3. Letters ' $N$ ' and 'E' respectively refer to 'no exposure' or 'exposure' at each of the first and second conditioning treatments.

Flight responses

Flight landing responses by each treatment of wasps were tested in a wind tunnel. A wind tunnel (450 $\mathrm{mm} \times$ $450 \mathrm{~mm} \times 850 \mathrm{~mm}$ ) made of acrylic resin was placed in a hood in which clean air was continuously supplied. Air in the wind tunnel was ventilated by a fan at the rate of $\sim 8001 \cdot \mathrm{min}^{-1}\left(\sim 50 \mathrm{~mm} \cdot \mathrm{s}^{-1}\right)$ and exhausted to outside of the room. For the release of wasps, a female A. gifuensis was collected in a plastic tube (6 mm i.d., $30 \mathrm{~mm}$ length) and the tube was individually placed on the platform. The number of individuals having oriented flight and landing on the target, which was placed $25 \mathrm{~cm}$ away upwind from the release point, in 3 min was counted. Experiments were conducted in a climate controlled room $\left(22 \pm 2^{\circ} \mathrm{C}, 20-40 \% \mathrm{RH}\right)$.

\section{Experiment 1}

The responses by wasps with different conditioning treatments (N-N, N-E, E-N and E-E) to four different target plants, wheat plants infested with 100 alate adults of $S$. akebiae for 3 days (wheat-PHC), undamaged wheat plants (wheat-UDP), broad bean plants infested with 50 adults of A. pisum for 3 days (bean-PHC) or undamaged broad bean (bean-UDP), were tested. Five replicates using different plant pots were conducted on different days in each target treatment, and each replicate consists of 24 trials of individual wasps (120 wasps in total were used in each combination of targets and wasp treatments). Each individual wasp was used only once.

\section{Experiment 2}

The responses by wasps with different conditioning treatments (N-N, N-E, E-N and E-E) to paper disk dummies $(70 \mathrm{~mm} \varnothing)$ of different colours, white or green, were tested. The relative reflectance spectra were measured for colours of each dummy using a spectrometer (HSU-100S, Asahi Spectra Co., Ltd; Fig. 2). Three replicates were conducted on different days using each colour of dummy and each replicate consisted of 12 trials of individual wasps (36 wasps in total were used in each combination of targets and wasp treatments). Each individual wasp was used only once. 


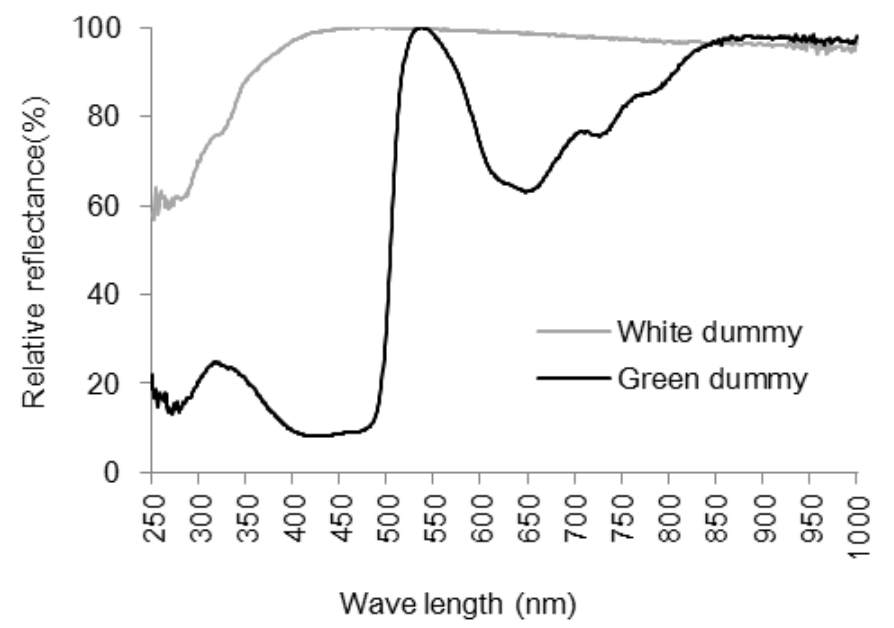

Fig. 2 Relative reflectance spectra of paper dummies.

Olfactory preferences

An olfactometer whose main arena was the Petri dish was used to test the olfactory preferences of female $A$. gifuensis wasps (Fig. 3). A Petri dish and odour source containers (W $150 \mathrm{~mm} \times$ D $200 \mathrm{~mm} \times \mathrm{H} 300 \mathrm{~mm}$ ) made of acrylic resin were connected by facing holes $(25 \mathrm{~mm} \varnothing)$ previously perforated on both the bottom of the odour source containers and the top of the Petri dish. A pot of UDP was put in one of the odour source containers, and that of the PHC was put in the other. The holes on odour source containers were covered with trap tubes (upside-down 50-ml plastic centrifuge tubes) whose side surface had openings $(40 \mathrm{~mm} \times 65 \mathrm{~mm})$ covered with nylon mesh so that the odour could pass through. For the release of wasps to the choice arena of the Petri dish, 30 wasps were collected in a plastic tube $(6 \mathrm{~mm} \varnothing \times 30 \mathrm{~mm})$, and it was placed on the hole at the bottom of the Petri dish. After $30 \mathrm{~min}$, the wasps in the trap tubes on each side of the odour sources were counted. Six replicates with different odour sources were conducted (180 wasps in total were used in each combination of odour sources and wasp treatments). Each individual wasp was used only once. Preferences by wasps between UDP and PHC in both the wheat/S. akebiae system and the bean/A. pisum system were tested.

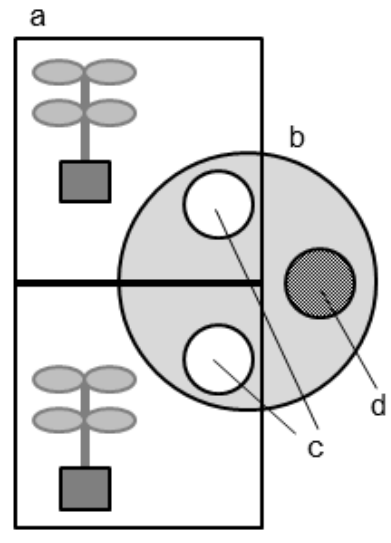

$<$ overhead view >

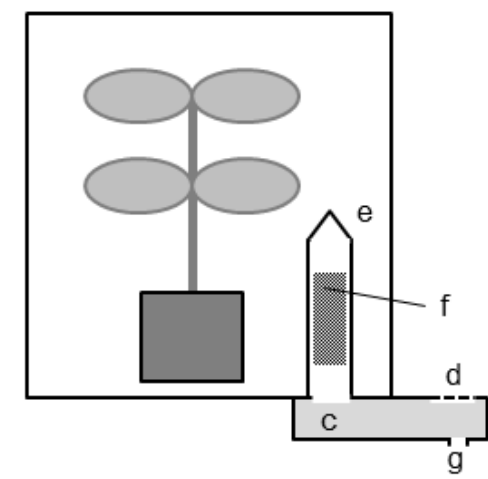

$<$ side view $>$

Fig. 3 Diagrams of the choice arena and the odour source containers used for olfactory preference tests. a: a pair of odour source containers made of acrylic resin $(200 \mathrm{~mm} \times 150 \mathrm{~mm} \times 300 \mathrm{~mm})$; b: choice arena in a Petri dish; c: holes $(25 \mathrm{~mm}$ $\varnothing, 70 \mathrm{~mm}$ distance between each centre) on the bottom of the container and top of the dish; d: air outlet hole (25 mm $\varnothing)$ covered with steel mesh; e: trap tube; f: opening covered with nylon mesh $(40 \mathrm{~mm} \times 65 \mathrm{~mm})$; and g: a hole for wasp release. 
Statistics

In Experiment 1 for flight responses, the proportion of wasps that landed on the source in each replicate $(\mathrm{N}=$ 5) was normalized using an arcsine square root transformation. The normality and homoscedasticity of the transformed data were confirmed using Kolmogorov-Smirnov and Bartlett tests, respectively. The proportions were analysed using a one-way ANOVA (treatments of wasps) followed by Tukey's HSD test using R (ver. 3.2.0; (R Core Team 2015) in each target. In Experiment 2, the statistical differences were examined between the total number of landed female wasps with oviposition experience and those without oviposition experience in experiments with each target using Fisher's exact test. The olfactory preference was statistically evaluated using a replicated $G$-test (Sokal and Rohlf 2012).

\section{Results}

Flight responses

Experiment 1

For all of the targets except for bean-PHC, landing rates of wasps that had host-encounter experiences (i.e., $\mathrm{N}-\mathrm{E}$ and E-E) were higher than those of wasps without host-encounters (i.e., N-N and E-N; Fig. 4a, 4b and 4d). Differences between landing rates of N-E and E-E wasps were not observed on those targets. For the bean-PHC target, the landing rate of E-E wasps was higher than those of the other conditioning treatments (Fig. 4c). The landing rates of E-N wasps to UDP targets were higher than those of N-N wasps on both wheat- and bean-UDP targets. The difference between the landing rates of $\mathrm{N}-\mathrm{E}$ and $\mathrm{E}-\mathrm{E}$ wasps was not observed on these UDP targets.

Experiment 2

Wasps did not show flight responses to the white dummy irrespective of experiences. One N-N wasp landed while the other 35 did not, and none of the 36 N-E, E-N and E-E wasps landed (Table 1). An increase in wasp landings on the green dummy after host-encounter experiences was observed for the wasps that had been kept in empty cages in early adult period. Six N-N wasps landed while 30 did not, and 23 N-E wasps landed while 13 did not (Fisher's exact test: P < 0.001). An increase in wasp landings on the green dummy after host-encounter experiences was seen for the wasps that had been exposed to undamaged wheat plants in the early adult period. Eight E-N wasps landed while 28 did not, and 28 N-E wasps landed while 8 did not (Fisher's exact test: $\mathrm{P}<0.001$; Table 1).

\section{Olfactory preferences}

Wasps that had been kept in empty cages in the early adult period did not show a preference between the W-UDP and the W-PHC, when they were either oviposition-naïve [N-N; W-UDP 51, W-PHC 45; P(Gp) = 0.540, P(Gh) < 0.001] or oviposition-experienced [N-E; W-UDP 42, W-PHC 39; P(Gp) = 0.739, P(Gh) = 0.024] (Fig. 5a). Wasps that had been exposed to the undamaged wheat plants in the early adult period did not show preferences between the W-UDP and the W-PHC when they were oviposition-naïve [E-N; W-UDP 44, W-PHC 45; P(Gp) $=0.916$, P(Gh) $=0.761$ ], but they preferred the W-PHC to the W-UDP when they were oviposition-experienced [E-E; W-UDP 40, W-PHC 66; P(Gp) = 0.011, $\mathrm{P}(\mathrm{Gh})=0.092$ ] (Fig. 5a). Wasps that had been kept in empty cages in the early adult period preferred the B-UDP to the B-PHC when they were either oviposition-naïve [N-N; B-UDP 59, B-PHC 36; P(Gp) $=0.018, \mathrm{P}(\mathrm{Gh})=0.104$ ], or oviposition-experienced [N-E; B-UDP 45, B-PHC 28; $\mathrm{P}(\mathrm{Gp})=0.046, \mathrm{P}(\mathrm{Gh})=0.051$ ] (Fig. 5b). Wasps that had been exposed to the W-UDP in the early adult period preferred the B-UDP to the B-PHC when they were oviposition-naïve [E-N; B-UDP 66, B-PHC 40; $\mathrm{P}(\mathrm{Gp})=0.011, \mathrm{P}(\mathrm{Gh})=0.871$ ], but not when they were oviposition-experienced [E-E; B-UDP 45, B-PHC 51; P(Gp) = 0.540, $\mathrm{P}(\mathrm{Gh})=0.122$ ] (Fig. 5b). 
(a) Wheat - PHC

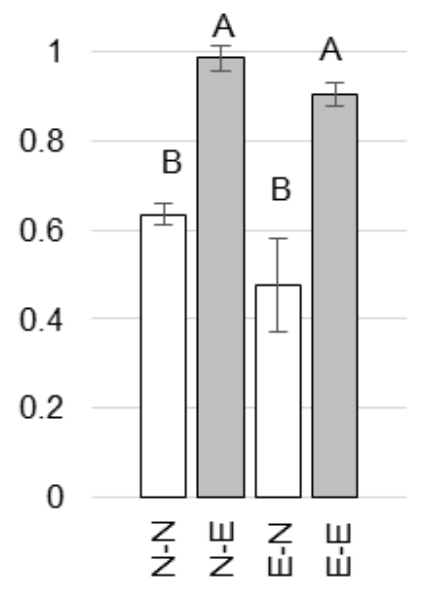

Treatment of wasps

(c) Broad Bean - PHC

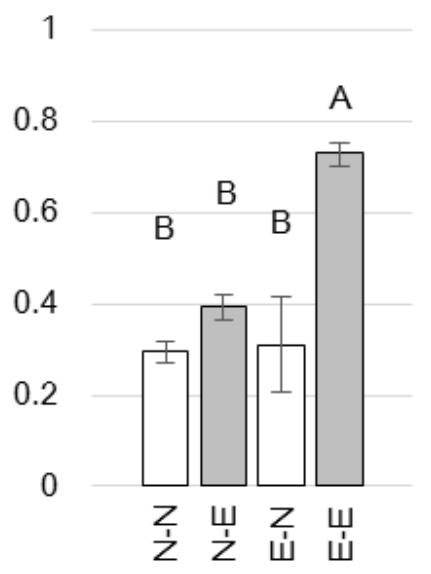

Treatment of wasps (b) Wheat - UDP

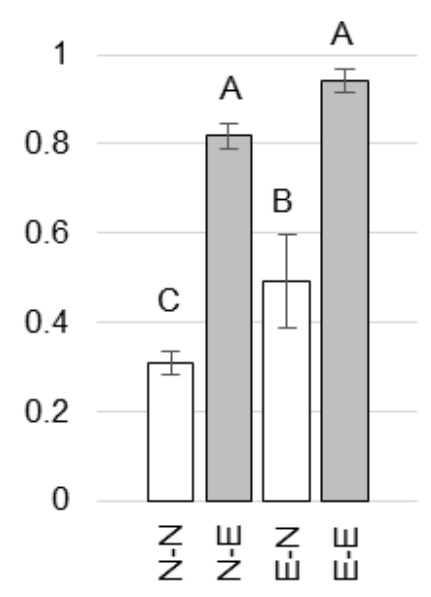

(d) Broad Bean - UDP

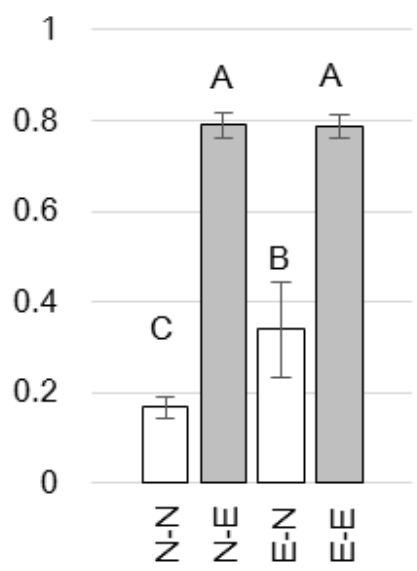

Treatment of wasps
Fig. 4 Proportion (mean \pm SE) of female $A$. gifuensis with different conditioning treatments that landed on the target in the wind tunnel assays. The numbers of test wasps were 24 par replicate and 120 in total in each combination of targets and wasp treatments. Letters ' $\mathrm{N}$ ' and ' $\mathrm{E}$ ' refer to 'no exposure' or 'exposure', respectively, at each of the first and second conditioning treatments. Columns with different letters are significantly different (Tukey's HSD test, $\alpha<0.05$ ). a: plant-host complex in the wheat system (wheat-PHC) b: undamaged plants in the wheat system (wheat-UDP) c: plant-host complex in the broad bean system (bean-PHC) d: undamaged plants in the broad bean system (broad bean-UDP).

Table 1 Flight responses of Aphidius gifuensis to paper dummies (The numbers of test wasps were 12 par replicate and 36 in total).

\begin{tabular}{llll}
\hline \multirow{2}{*}{ Target } & conditioning & \\
\cline { 3 - 4 } (dummy colour) & of wasps & orientated flight & landing on the target \\
\cline { 3 - 4 } White & N-N & 4 & 1 \\
& N-E & 5 & 0 \\
& E-N & 0 & 0 \\
& E-E & 1 & 0 \\
\hline Green & N-N & 8 & 6 \\
& N-E & 25 & 23 \\
& E-N & 12 & 8 \\
& E-E & 28 & 28 \\
\hline
\end{tabular}


(a) Wheat

\section{$P G p$}

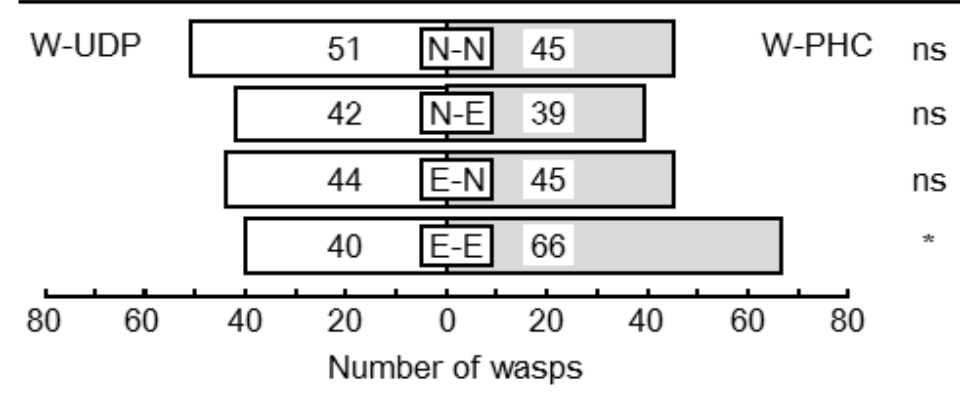

(b) Broad bean

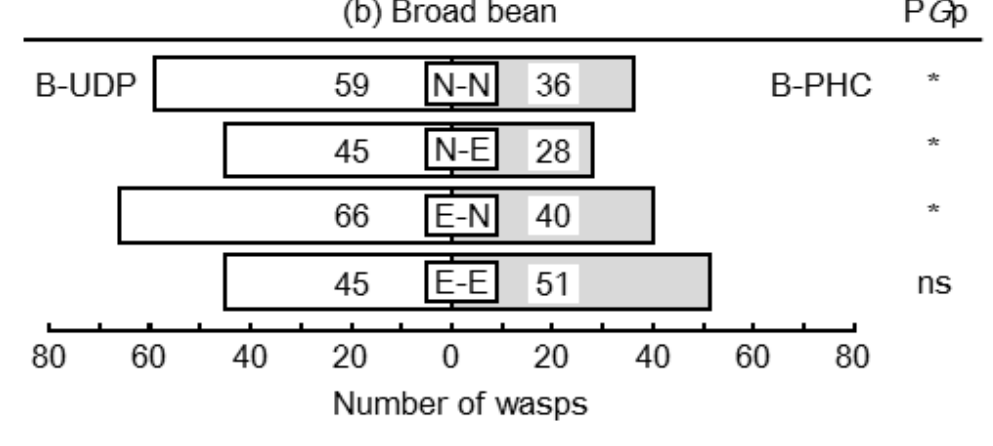

Fig. 5 Olfactory preferences of female A. gifuensis exposed to different conditioning treatments between undamaged plants (UDPs) and plant-host complexes (PHCs) in either natal wheat or non-natal broad bean systems. Letters ' $N$ ' and ' $E$ ' refer to 'no exposure' or 'exposure', respectively, at each of the first and second conditioning treatments. $* \mathrm{P}(G \mathrm{p})<0.05$, ns not significant (replicated $G$-test).

\section{Discussion}

The flight response of female A. gifuensis wasps to natal wheat-PHCs and natal wheat-UDPs increased after host encounter experience (exposure of wasps to the natal wheat PHCs). Because the increase was also found in the response to the non-natal bean-UDPs, the cues involved in the increase of responses were not stimuli specific to the natal habitats. Similarly the increase was also found in responses to green coloured dummies, whereas it was not found with white coloured dummies. The relative reflectance spectra of the white colour dummy showed a high reflectance rate at the broad wave length area from $320 \mathrm{~nm}$ to $700 \mathrm{~nm}$ and that of the green dummy showed the characteristic peak at $\sim 550 \mathrm{~nm}$, indicating green colour. The responses of wasps to the different coloured dummies suggested that the cues involved in the increase in landing rates includes general visual cues of plants such as colour or brightness.

In the test of olfactory preference, E-E wasps preferred odours from the wheat-PHC over those of the wheat-UDP, but $\mathrm{N}-\mathrm{E}$ wasps did not. The experience of UDP in the natal habitat followed by exposure to natal PHCs reinforced the response to olfactory stimuli from natal PHCs independent of the visual stimuli. In a supplemental experiment, encounters with hosts only, without plants, did not reinforce olfactory responses to natal PHCs [W-UDP 37, W-PHC 49; $\mathrm{P}(\mathrm{Gp})=0.195, \mathrm{P}(\mathrm{Gh})=0.141$; supplemental figure $)$. These results suggested that the reinforcement of olfactory response was not explained by a sensitization to general olfactory stimuli. In other words, the involvement of associative learning with stimuli from plants was suggested. In addition, differences between flight responses to wheat-PHCs by N-E wasps and E-E wasps were not observed. These results suggest that the associative learning with olfactory stimuli did not strongly influence the flight responses by wasps in the wind tunnel and that visual stimuli were primal cues.

Interestingly, the flight responses of female A. gifuensis wasps to the non-natal bean-PHCs increased after exposure to the natal PHCs in the E-E conditioning treatment but did not increase in the N-E conditioning treatment. Thus, the experience in the natal UDP prior to the host-encounter in natal PHCs influenced the response after the host-encounter experience. An olfactory preference for bean-UDPs to bean-PHCs was found in N-N, N-E and E-N wasps. This suggested that odours from the bean-PHCs can decrease the host locating efficiency of A. gifuensis female wasps in 
broad bean habitats. Female A. gifuensis wasps may innately repel odours from the bean-PHCs. However, E-E wasps showed different olfactory preferences and the negative olfactory response to bean-PHCs disappeared in E-E wasps. Thus, these results suggested that the early exposure to natal habitat plants modified later learning and response to visual cue from potential non-natal habitat plants, and olfactory learning might be involved in the modification. The negative olfactory information might have suppressed the response to non-specific visual stimuli and E-E wasps have responded to bean-PHCs in the wind tunnel assay because of the removal of the suppression.

Landing rates on both natal and non-natal UDPs increased with natal UDP experiences alone. Thus, the landing rates of E-N wasps were higher than those of N-N in responses to natal and non-natal UDPs. The difference between N-N and E-N wasps was not found in their responses to PHC targets, suggesting that the stimuli involved in the reinforcement of the response to UDPs by the experience of natal-UDPs are different from the general visual stimuli involved in the reinforcement by host-encounters in natal-PHCs. Another generalist aphid parasitoid, A. colemani, acquired an olfactory preference for chemical compounds, which had been adsorbed on the mummy surface, that they experienced during emergence (Storeck et al. 2000; van Emden et al. 2008). The details of the cues and timing involved in the reinforcement by exposure to natal UDPs in A. gifuensis need to be determined.

Despite the ability of E-E wasps to discriminate odours from PHCs in wheat habitats, they also responded to the natal wheat-UDPs and non-natal bean-UDPs in the wind tunnel. This suggested that the response to non-specific visual stimuli comes before that of the specific olfactory stimuli in the host location process. In the periods shortly after host-encounter experiences in the natal habitat, other available host individuals are likely to be found nearby. It would be beneficial to search on neighbouring plants using visual stimuli that provide a higher recognition speed. Additionally, the conditioning treatments of E-E wasps represent a situation in which wasps remained on natal habitat plants from their emergence until the host-encounter. While searching in a microhabitat, visual stimuli, such as green colour and the brightness of objects, are not useful cues to find hosts. In this situation, wasps probably use learned information from the specific olfactory stimuli as cues for locating the precise host position.

It might be generally assumed that learning in a particular habitat makes foragers specialized in the habitat. In female A. gifuensis, however, the stimuli involved in the reinforcement were not specific to the stimuli from the habitat. In previous studies, A. gifuensis did not show olfactory preferences among tobacco plants infested by M. persicae, oilseed rape infested by Lipaphis erysimi Kaltenbach (Hemiptera: Aphididae) or oilseed rape infested by M. persicae (Yang et al. 2009a). Responses specific to the natal habitat were also not found in the study. In habitat generalists, possessing the ability to respond to a broad range of cues from available resources can be adaptive. Experienced A. gifuensis wasps that are moving to a non-natal habitat may keep the ability to respond to a broad range of stimuli, even if they have experienced oviposition on hosts in the natal habitat. The volatiles induced in broad bean plants by A. pisum aphids attract the specialist parasitic wasp A. ervi (Takemoto et al. 2012), but they repelled inexperienced A. gifuensis in this study. The decrease in the foraging efficiency of generalists may be an adaptive trait of this specialist aphid, as along with the induction of physiological changes that increase resource quality for the conspecific aphids (Takemoto et al. 2013). Although the efficiency to use particular information in the generalist A. gifuensis can be lower than that in specialist parasitoids, the reinforcement of responses to cues not specific to the natal habitat would be beneficial to ensure the availability of cues from other non-natal habitat plants.

This study showed that early experience of exposure to natal habitat plants was involved in habitat selection process of the habitat generalist parasitoid A. gifuensis, and the experience resulted in an increase of receptivity to cues from potential non-natal habitat plants. The mechanisms in the increase of receptivity would be a cross modal effect that the early exposure to natal habitat plants modified later learning and response to visual cue from potential non-natal habitat plants, and olfactory learning might be involved in the modification. Modification of preference for visual stimuli in the presence of olfactory stimuli has been found in the parasitoid Cotesia vestalis Haliday (Hymenoptera: Braconidae) in a physiological state-dependent manner (Uefune et al. 2012) and in the swallowtail butterfly Papilio xuthus Linnaeus (Lepidoptera: Papilionidae) in a sex-dependent manner (Yoshida et al. 2015). Present study for the first time shows modification of response to visual stimuli in the presence of olfactory stimuli in an experience-dependent manner. Integration of multimodal stimuli provides advantages for communication through strategic benefits as increase of salience of the signal or efficacy benefits as increase of redundancy of the signal (Stevens 2013). Since a key benefit of redundancy is in getting the message across more effectively rather than altering the message itself (Stevens 2013), the cross modal effect of the natal experience altering message of visual stimuli in present study would provide an advantage of a strategic benefit in habitat selection or searching behaviour in A. gifuensis. Future investigations about detailed mechanisms of the cross modal integration in the response to novel habitat cues are required for understanding of information use in habitat selection behaviour and dispersal in this species. 


\section{Acknowledgments}

The author thanks Izumi Ohta for providing insect colonies, Mantaro Hironaka for his help in the measurement of relative reflectance of paper dummies, and the Research Institute of Green Science and Technology, Shizuoka University, for providing research facilities. This research was supported (in part) by The Japan Health Foundation and a Grant for Environmental Research Projects from The Sumitomo Foundation.

\section{References}

Blande JD, Pickett JA, Poppy GM (2007) A Comparison of Semiochemically Mediated Interactions Involving Specialist and Generalist Brassica-feeding Aphids and the Braconid Parasitoid Diaeretiella rapae. J Chem Ecol 33:767-779. doi: 10.1007/s10886-007-9264-7

Chittka L, Raine NE (2006) Recognition of flowers by pollinators. Curr Opin Plant Biol 9:428-435. doi: 10.1016/j.pbi.2006.05.002

Davis JM, Stamps JA (2004) The effect of natal experience on habitat preferences. Trends Ecol Evol 19:411-416. doi: 10.1016/j.tree.2004.04.006

Dobson HEM (1994) Floral volatiles and in insect biology. In: Bernays E (ed) Insect-plant interactions. CRC Press, London, pp 47-81

Dötterl S, Vereecken NJ (2010) The chemical ecology and evolution of bee-flower interactions: a review and perspectives. Can J Zool 88:668-697. doi: 10.1139/Z10-031

Godfray HCJ (1993) Parasitoids: Behavioral and Evolutionary Ecology. Princeton University Press, Princeton

Nakahira K, Takada Y, Teramoto T, Kagoshima K, Takagi M (2012) Control of potato aphids by the addition of barley strips in potato fields: a successful example of vegetation management. Biocontrol Sci Technol 22:1155-1165. doi: $10.1080 / 09583157.2012 .715627$

Ohta I, Honda K (2010) Use of Sitobion akebiae (Hemiptera: Aphididae) as an alternative host aphid for a banker-plant system using an indigenous parasitoid, Aphidius gifuensis (Hymenoptera: Braconidae). Appl Entomol Zool 45:233-238. doi: 10.1303/aez.2010.233

Ohta I, Ohtaishi M (2004) Fertility, longevity and intrinsic rate of increase of Aphidius gifuensis Ashmead (Hymenoptera: Braconidae) on the green peach aphid, Myzus persicae (Sulzer) (Homoptera: Aphididae). Appl Entomol Zool 39:113-117. doi: 10.1303/aez.2004.113

Ohta I, Ohtaishi M (2005) Effectiveness of Aphidius gifuensis ashmead (Hymenoptera: Braconidae) on the population control of Myzus persicae (Sulzer) (Homoptera: Aphididae) in small experimental greenhouses. Japanese J Appl Entomol Zool 49:78-82. doi: 10.1303/jjaez.2005.78

R Core Team (2015) R; A language and environment for statistical computing.

Sokal RR, Rohlf FJ (2012) Biometry: the principles and practice of statistics in biological research, 4th edn. W. H. Freeman and Company, New York

Stevens M (2013) Sensory Ecology, Behaviour, and Evolution. Oxford University Press, oxford

Storeck A, Poppy G, van Emden H, Powell W (2000) The role of plant chemical cues in determining host preference in the generalist aphid parasitoid Aphidius colemani. Entomol Exp Appl 97:41-46. doi: 10.1046/j.1570-7458.2000.00714.x

Takada H (1992) Aphid parasitoids as biological control agents to vector aphids of papaya ring spot virus and banana bunchy top virus. FFTC Tech Bull 132:1-11.

Takada H (2002) Parasitoids (Hymenoptera : Braconidae, Aphidiinae; Aphelinidae) of four principal pest aphids (Homoptera : Aphididae) on greenhouse vegetable crops in Japan. Appl Entomol Zool 37:237-249. doi: 10.1303/aez.2002.237

Takemoto H, Powell W, Pickett J, Kainoh Y, Takabayashi J (2012) Two-step learning involved in acquiring olfactory preferences for plant volatiles by parasitic wasps. Anim Behav 83:1491-1496. doi: 10.1016/j.anbehav.2012.03.023

Takemoto H, Uefune M, Ozawa R, Arimura G, Takabayashi J (2013) Previous infestation of pea aphids Acyrthosiphon pisum on broad bean plants resulted in the increased performance of conspecific nymphs on the plants. J Plant Interact 8:370-374. doi: 10.1080/17429145.2013.786792

Turlings TCJ, Wäckers FL, Vet LEM, Lewis WJ, Tumlinson JH (1993) learning of host-finding cues by Hymenopterous parasitoids. insect Learn. 51-78.

Uefune M, Kugimiya S, Shimoda T, Takabayashi J (2012) Starvation and herbivore-induced plant volatiles affect the color preferences of parasitic wasps. BioControl 58:187-193. doi: 10.1007/s10526-012-9483-x

van Emden H, Eletherianos I, Rose J, Douloumpaka S, Pettersson J (2002) Aphid parasitoid detects that an alien plant was present nearby during their development. Physiol Enthomology 27:199-205.

van Emden HF, Storeck AP, Douloumpaka S, Eletherianos I, Poppy GM, Powell W (2008) Plant chemistry and aphid 
parasitoids (Hymenoptera: Braconidae): Imprinting and memory. Eur J Entomol 105:477-483. doi: 10.14411/eje.2008.061

Vet LEM, Dicke M (1992) Ecology of Infochemical Use by Natural Enemies in a Tritrophic Context. Annu Rev Entomol 37:141-172. doi: 10.1146/annurev.en.37.010192.001041

Vet LEM, Lewis WJ, Cardé RT (1995) Parasitoid Foraging and Learning. In: Chemical Ecology of Insects 2. Springer US, Boston, MA, pp 65-101

Wei J, Li T, Kuang R, Wang Y, Yin T, Wu X, Zou L, Zhao W, Cao J, Deng J (2003) Mass Rearing of Aphidius gifuensis (Hymenoptera: Aphidiidae) for Biological Control of Myzus persicae (Homoptera: Aphididae). Biocontrol Sci Technol 13:87-97. doi: 10.1080/0958315021000054412

Yang S, Xu R, Yang S, Kuang R (2009a) Olfactory responses of Aphidius gifuensis to odors of host plants and aphid-plant complexes. Insect Sci 503-510. doi: 10.1111/j.1744-7917.2009.01282.x

Yang S, Yang SY, Zhang CP, Wei J-N, Kuanga RP (2009b) Population dynamics of Myzus persicae on tobacco in Yunnan Province, China, before and after augmentative releases of Aphidius gifuensis. Biocontrol Sci Technol 19:219-228. doi: 10.1080/09583150802696525

Yoshida M, Itoh Y, Ômura H, Arikawa K, Kinoshita M (2015) Plant scents modify innate colour preference in foraging swallowtail butterflies. Biol Lett 11:20150390. doi: 10.1098/rsbl.2015.0390

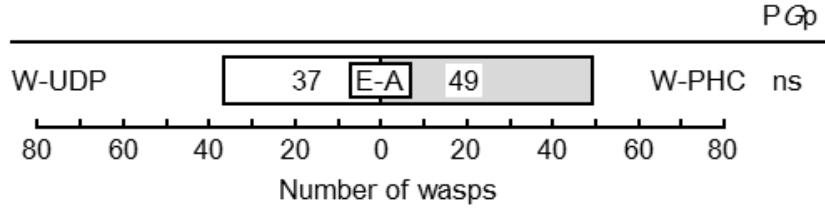

Supplemental figure Olfactory preferences of female A. gifuensis that had been exposed to wheat-UDP and sequentially to host aphids only (indicated as E-A in the figure) between undamaged plants (UDP) and plant-host complex (PHC) in the wheat system. ns indicates not significant (replicated $G$-test) 